\title{
Effect of Emeriamine, an Inhibitor of Fatty Acid Oxidation, on Metabolic Fate of a Geometrical Isomer of Linoleic Acid in Perfused Rat Liver
}

\author{
Nobuhiro FukUda, ${ }^{1, *}$ Masanori FuKUi, ${ }^{1}$ Yasuko KaI, ${ }^{1}$ \\ Anura P. JAYASOORIYA, ${ }^{1}$ Masanobu SAKono, ${ }^{1}$ \\ Hideo MAEDA, ${ }^{2}$ Takashi IdE ${ }^{3}$ \\ and Kyosuke Yамамото ${ }^{4}$ \\ ${ }^{1}$ Department of Biological Resource Sciences, Faculty of Agriculture, \\ Miyazaki University, Miyazaki 889-2192, Japan \\ ${ }^{2}$ Faculty of Health and Living Sciences, Naruto University of Education, \\ Naruto 772-8502, Japan \\ ${ }^{3}$ Laboratory of Nutrition Biochemistry, National Food Research Institute, \\ Ministry of Agriculture, Fisheries and Forestry, Tsukuba 305-8642 \\ ${ }^{4}$ Department of Internal Medicine, Saga Medical University School of Medicine, \\ Saga 849-8501, Japan \\ (Received March 23, 1998)
}

\begin{abstract}
Summary To estimate the relative significance of exogenous vs. endogenous fatty acids in increasing hepatic triacylglycerol secretion following an inhibition of fatty acid oxidation by emeriamine, livers from 2-d-fasting rats were perfused with or without an inhibitor in the presence of a geometrical isomer of linoleate (linolelaidic acid, trans, trans-9,12octadecadienoic acid). Emeriamine added to the perfusion medium at $2 \mathrm{~h}$ of the recirculating perfusion period caused immediate and complete cessation of ketone body production while it increased triacylglycerol and cholesterol secretion by the liver without affecting uptake of exogenous linolelaidic acid. The increase in the triacylglycerol secretion by emeriamine was accompanied by a marked increase in the proportion of linolelaidic acid in this lipid molecule in the perfusate and in the liver. The calculated amounts of exogenous linolelaidate, compared with those of endogenous fatty acids in the secretory triacylglycerol, suggested that the former compared with the latter contributes more to the drug-mediated increase in triacylglycerol secretion. This drug caused a marked reduction of mitochondrial carnitine palmitoyltransferase activity in perfused liver. These results suggest that a blockade of fatty acid oxidation by emeriamine, through an inhibition of carnitine palmitoyltransferase, diverts predominantly the exogenous free fatty acids from oxidation to the esterification pathway and subsequently stimulates the synthesis and secretion
\end{abstract}

\footnotetext{
* To whom correspondence should be addressed.
} 
of triacylglycerol-rich lipoproteins.

Key Words fatty acid oxidation inhibitor, emeriamine, ketone body production, triacylglycerol secretion, perfused rat liver

It is considered that the fatty acid oxidation rate is a critical determinant in regulating hepatic triacylglycerol synthesis and secretion in the rat $(1,2)$. Our previous studies using an inhibitor of mitochondrial fatty acid oxidation, emeriamine [(R)-3-amino-4-trimethylaminobutyric acid], support this hypothesis. This drug is a specific and potent inhibitor of carnitine palmitoyltransferase I and thus reduces fatty acid oxidation rate through the retardation of the transportation of fatty acids across the mitochondrial membrane (3-5). We previously reported that the inhibition of fatty acid oxidation by emeriamine increases triacylglycerol and cholesterol secretion in isolated liver perfused with oleic acid substrate in 2-d-fasting rats (6). Furthermore, the oral administration of emeriamine caused hypertriglyceridemia and an accumulation of hepatic triacylglycerol in fasting rats $(7,8)$. However, in the previous liver perfusion study we did not assess the relative contribution of endogenous and exogenous fatty acids to the emeriamine-induced stimulation of triacylglycerol secretion.

In this experiment, we therefore compared the metabolism of a geometrical isomer of linoleate (linolelaidate, trans,trans-9,12-octadecadienoic acid) between perfused rat liver treated with this inhibitor and without it. This geometrical isomer of linoleate was chosen as a tracer to examine the metabolic fate of exogenous fatty acid in perfused rat liver because of its rare occurrence in nature, and it is distinguishable from other endogenous fatty acids and quantifiable by gas-liquid chromatography on Silar 10 C column $(9,10)$.

\section{MATERIALS AND METHODS}

Materials. Linolelaidic acid and palmitoyl-CoA were purchased from Sigma Chemical, St. Louis, MO. Bovine serum albumin Fraction V and $\beta$-hydroxybutyrate dehydrogenase were obtained from Boehringer Mannheim $\mathrm{GmbH}$ (Mannheim, Germany). Emeriamine [(R)-3-amino-4-trimethylaminobutyric acid] (a deacylation derivative of emericedin that was produced by Emericella quadrilineata IFO 5859) was provided by Takeda Chemical Industries (Osaka, Japan). All other chemicals used were of analytical grade.

Liver perfusion. Male Sprague-Dawley rats weighing an average $280 \mathrm{~g}$ (Kyudo, Kumamoto, Japan) were given a pellet-stocked chow (Type CE-2, Clea Japan, Tokyo, Japan) and were acclimated for several days in a temperaturecontrolled room $\left(22-24^{\circ} \mathrm{C}\right.$, light on $\left.07: 00-19: 00\right)$. They then received only water for $2 \mathrm{~d}$ before the day of liver perfusion experiments. On the day of experiments, rat livers were isolated under Nembutal anesthesia at around 09:00 and perfused 
with $120 \mathrm{~mL}$ of recirculating Krebs-Henseleit buffer ( $\mathrm{pH} 7.4$ ), which contained $0.1 \%$ glucose, $1.5 \%$ fatty acid free bovine serum albumin, and $25 \%(\mathrm{w} / \mathrm{v})$ washed bovine erythrocytes, at a rate of $20 \mathrm{~mL} / \mathrm{min}$ in a perfusion apparatus thermostatistically controlled at $37^{\circ} \mathrm{C}$. At the beginning of recirculation, $5 \mathrm{~mL}$ of $20 \mathrm{~mm}$ potassium linolelaidate (100 $\mu \mathrm{mol}$, trans,trans-9,12-octadecadienoic acid) was added, and the same solution was continuously infused at the rate of $4.5 \mathrm{~mL} / \mathrm{h}(90 \mu \mathrm{mol} / \mathrm{h})$. Emeriamine $(2 \mu \mathrm{mol} / \mathrm{mL})$ dissolved in saline was added $2 \mathrm{~h}$ after the initiation of recirculating liver perfusion. Another $1 \mu \mathrm{mol}$ of inhibitor was added at $3 \mathrm{~h}$ in view of its possible metabolism by the liver (4). The perfusion was continued for a total of $4 \mathrm{~h}$. Twenty milliliters of perfusate was removed at every hour of the experimental period for analyses of lipids and ketone bodies, and the same volume of the fresh perfusion medium was added at each removal. The perfusion equipment and procedures were those previously described in detail $(11,12)$.

Chemical analyses. After termination of liver perfusion, a portion of liver was homogenized in 9 volumes of $0.25 \mathrm{~m}$ sucrose solution. The homogenate was centrifuged at $1,000 \times g$ for $10 \mathrm{~min}$ to sediment nuclei and cell debris, and the supernatant was then centrifuged at $12,500 \times g$ for 20 min to sediment the mitochondria (13); the mitochondria were resuspended in a solution containing $0.3 \mathrm{~m}$ mannitol, $10 \mathrm{~mm}$ HEPES, and $0.1 \mathrm{~mm}$ EGTA and stored at $-45^{\circ} \mathrm{C}$ until the analysis was done. Carnitine palmitoyltransferase activity in mitochondria was assayed in $58 \mathrm{~mm}$ Tris- $\mathrm{HCl}$ (pH 8.0) containing $1.25 \mathrm{~mm}$ EDTA, $0.25 \mathrm{~mm}$ DTNB, $37.5 \mathrm{~mm}$ palmitoyl-CoA, and $1.25 \mathrm{~mm}$ L-carnitine according to the method of Markwell et al (14). The activity obtained in the absence of L-carnitine was used as a blank. Mitochondrial protein was analyzed by the method of Lowry et al (15). The lipids of erythrocyte-free perfusate and postperfused liver were extracted and purified according to the method of Folch et al (16). Triacylglycerol, cholesterol, phospholipid, and free fatty acid contents in the lipid extract were measured as described elsewhere $(9,10)$. Concentrations of $\beta$-hydroxybutyrate and acetoacetate in a deproteinized sample of liver perfusate were measured enzymatically as reported previously and expressed as total ketone body production (9-12). Triacylglycerol, phospholipid and cholesterol ester in perfusate, and postperfused liver were separated by silica gel $60 \mathrm{G}$ thin-layer chromatography by using a solvent system of $n$-hexane : diethyl ether: glacial acetic acid, $80: 20: 1, \mathrm{v} / \mathrm{v} / \mathrm{v}$, and their fatty acid methyl esters were analyzed by gas-liquid chromatography on Silar $10 \mathrm{C}$ column $(9,10)$.

Statistical analyses. Data were expressed as mean $\pm \mathrm{SE}$, and the statistical significance of the difference of means was evaluated by Student's $t$-test at the level of $p<0.05$ (17).

\section{RESULTS}

\section{Hepatic lipid concentrations and carnitine palmitoyltransferase activity}

The average body weight of animals on the day of experiment was $303 \pm 3 \mathrm{~g}$. 
Table 1. Uptake of exogenous linolelaidic acid substrate, hepatic lipid concentration, and its mitochondrial carnitine palmitoyltransferase activity.

\begin{tabular}{lrr}
\hline & $\begin{array}{c}\text { Control } \\
(5)\end{array}$ & $\begin{array}{c}\text { Emeriamine } \\
\text { at } 2 \mathrm{~h}(6)\end{array}$ \\
\hline Liver lipid ( $\mu \mathrm{mol} / \mathrm{g}$ liver) & & \\
$\quad$ Triacylglycerol & $16.7 \pm 2.2$ & $20.4 \pm 4.9$ \\
$\quad$ Total cholesterol & $8.0 \pm 0.6$ & $8.2 \pm 0.5$ \\
$\quad$ Phospholipid & $27.1 \pm 5.5$ & $30.9 \pm 3.8$ \\
Carnitine palmitoyltransferase activity & & \\
$\quad$ (nmol CoA/min/mg protein) & $12.0 \pm 0.6$ & $0.6 \pm 0.1^{*}$ \\
\hline
\end{tabular}

Numbers in parentheses indicate the number of animals, and the results are expressed as mean \pm SE. * Significantly different from control group at $p<0.05$.

Emeriamine added to the perfusion medium at $2 \mathrm{~h}$ did not affect the relative weights of the livers measured at the end of a $4 \mathrm{~h}$ recirculation period $(2.8 \pm 0.1 \mathrm{~g} / 100 \mathrm{~g}$ body weight for control and drug-treated livers). Drug treatment was ineffective in modulating triacylglycerol, cholesterol, and phospholipid concentration in the liver (Table 1). Mitochondrial carnitine palmitoyltransferase activity was markedly lower in the drug-treated liver than in the control liver.

The amounts of exogenous linolelaidate taken up by control livers, which were calculated from the amounts infused and those remaining in the perfusate at 1,2 , 3 , and $4 \mathrm{~h}$ of the perfusion periods were $21.4 \pm 1.2,31.5 \pm 1.5,41.9 \pm 2.2$, and $51.1 \pm 2.2 \mu \mathrm{mol} / \mathrm{g}$ liver, and they were similar to those in the livers treated with emeriamine $(22.1 \pm 0.9,32.8 \pm 0.7,42.6 \pm 0.3$, and $51.4 \pm 0.9 \mu \mathrm{mol} / \mathrm{g}$ liver $)$. Therefore the possibility that emeriamine affects the hepatic uptake of exogenous fatty acid and thus modifies ketogenesis and lipid secretion could be disregarded.

\section{Ketone body production and lipid secretion}

Ketone bodies accumulated linearly in the perfusate of control livers during the entire recirculating period (Table 2). On the other hand, emeriamine caused immediate and almost complete cessation of ketone body production. The ratio of $\beta$-hydroxybutyrate to acetoacetate in the control livers gradually decreased during $4 \mathrm{~h}$ perfusion periods, while emeriamine treatment $2 \mathrm{~h}$ later caused a marked and rapid decrease in the ratio.

Only a limited amount of triacylglycerol was detected in the perfusates from control liver at $1 \mathrm{~h}$ of the recirculating period, and no cumulative increase in triacylglycerol secretion was observed in these livers during the rest of the experimental period. However, triacylglycerol began to accumulate in the perfusion medium soon after an addition of emeriamine at $2 \mathrm{~h}$, and the amounts of triacylglycerol secreted at 3 and $4 \mathrm{~h}$ of the experimental period were significantly higher in the drug-treated liver than in the control liver. 
Table 2. Effect of emeriamine on ketone body production, the ratio of $\beta$ hydroxybutyrate and acetoacetate, and the net secretion of triacylglycerol and cholesterol by the livers.

\begin{tabular}{|c|c|c|c|c|}
\hline & \multicolumn{4}{|c|}{ Hours } \\
\hline & 1 & 2 & 3 & 4 \\
\hline \multicolumn{5}{|c|}{ Ketone body production ( $\mu \mathrm{mol} / \mathrm{g}$ liver $)$} \\
\hline Control (5) & $50.5 \pm 4.8$ & $81.7 \pm 5.7$ & $106.9 \pm 6.0$ & $127.2 \pm 3.0$ \\
\hline Emeriamine at $2 \mathrm{~h}(6)$ & $48.2 \pm 2.0$ & $75.0 \pm 1.2$ & $69.7 \pm 1.8^{*}$ & $74.2 \pm 2.4^{*}$ \\
\hline \multicolumn{5}{|c|}{ Ratio of $\beta$-hydroxybutyrate: acetoacetate } \\
\hline Control (5) & $1.00 \pm 0.24$ & $0.65 \pm 0.12$ & $0.54 \pm 0.11$ & $0.53 \pm 0.10$ \\
\hline Emeriamine at $2 \mathrm{~h}(6)$ & $0.82 \pm 0.05$ & $0.68 \pm 0.05$ & $0.29 \pm 0.02 *$ & $0.26 \pm 0.02 *$ \\
\hline \multicolumn{5}{|c|}{ Triacylglycerol secretion ( $\mu \mathrm{mol} / \mathrm{g}$ liver $)$} \\
\hline Control (5) & $0.39 \pm 0.04$ & $0.37 \pm 0.03$ & $0.34 \pm 0.03$ & $0.38 \pm 0.04$ \\
\hline Emeriamine at $2 \mathrm{~h}(6)$ & $0.42 \pm 0.04$ & $0.43 \pm 0.04$ & $0.87 \pm 0.07 *$ & $1.69 \pm 0.07 *$ \\
\hline \multicolumn{5}{|c|}{ Exogenous linolelaidic acid in perfusate triacylglycerol-fatty acids (weight \%) } \\
\hline Control (5) & $25.9 \pm 1.0$ & $21.7 \pm 4.1$ & $17.8 \pm 2.3$ & $15.7 \pm 3.8$ \\
\hline Emeriamine at $2 \mathrm{~h}(6)$ & $21.9 \pm 2.2$ & $20.5 \pm 2.1$ & $41.9 \pm 1.2^{*}$ & $51.7 \pm 3.2^{*}$ \\
\hline \multicolumn{5}{|c|}{ Exogenous linolelaidic acid in perfusate triacylglycerol-fatty acids $(\mu \mathrm{mol} / \mathrm{g} \text { liver })^{1}$} \\
\hline Control (5) & $0.31 \pm 0.04$ & $0.26 \pm 0.06$ & $0.19 \pm 0.04$ & $0.18 \pm 0.04$ \\
\hline Emeriamine at $2 \mathrm{~h}(6)$ & $0.28 \pm 0.04$ & $0.26 \pm 0.04$ & $1.12 \pm 0.11^{*}$ & $2.60 \pm 0.11^{*}$ \\
\hline \multicolumn{5}{|c|}{ Endogenous fatty acids in perfusate triacylglycerol-fatty acids $(\mu \mathrm{mol} / \mathrm{g} \mathrm{liver})^{2}$} \\
\hline Control (5) & $0.86 \pm 0.08$ & $0.87 \pm 0.02$ & $0.84 \pm 0.05$ & $0.97 \pm 0.07$ \\
\hline Emeriamine at $2 \mathrm{~h}(6)$ & $0.99 \pm 0.07$ & $1.03 \pm 0.11$ & $1.52 \pm 0.14^{*}$ & $2.48 \pm 0.26^{*}$ \\
\hline \multicolumn{5}{|c|}{ Cholesterol secretion $(\mu \mathrm{mol} / \mathrm{g}$ liver $)$} \\
\hline Control (5) & $0.19 \pm 0.02$ & $0.24 \pm 0.03$ & $0.28 \pm 0.02$ & $0.36 \pm 0.03$ \\
\hline Emeriamine at $2 \mathrm{~h}(6)$ & $0.19 \pm 0.02$ & $0.22 \pm 0.03$ & $0.38 \pm 0.02 *$ & $0.59 \pm 0.06^{*}$ \\
\hline
\end{tabular}

Numbers in parentheses indicate the number of animals, and the results are expressed as mean $\pm \mathrm{SE} .{ }^{*}$ Significantly different from control group at $p<0.05$.

${ }^{1}[\mu \mathrm{mol}$ of triacylglycerol $\times 3 \times$ percentage of exogenous linolelaidic acid in perfusate triacylglycerol $/ 100]$.

${ }^{2}[\mu$ mol of triacylglycerol $\times 3 \times$ percentage of endogenous fatty acids in perfusate triacylglycerol/100].

The proportion of linolelaidic acid in perfusate triacylglycerol at each time interval was determined by gas-liquid chromatography (Table 2). In perfusate obtained from control liver at $1 \mathrm{~h}$ of the perfusion period, the proportion of the transfatty acid in triacylglycerol was $26 \%$, and it gradually decreased to $16 \%$ at the end of perfusion. The difference between $1 \mathrm{~h}$ and $4 \mathrm{~h}$ being statistically significant at $p<0.05$. This result suggests that $74-84 \%$ of perfusate-triacylglycerol fatty acids are derived from endogenous origins in control livers during the entire experimental period. Emeriamine treatment at $2 \mathrm{~h}$ caused a marked increase in the proportion of exogenous transfatty acid in perfusate triacylglycerol. The increase in the value was already apparent at $3 \mathrm{~h}$ of the experimental period $(17.8 \%$ for the control and 
$41.9 \%$ for the drug-treated livers, the difference being statistically significant at $p<0.05$ ).

Based on the analysis by gas-liquid chromatography of fatty acids, the amounts of exogenous transfatty acid and endogenous fatty acids secreted as components of perfusate triacylglycerol were calculated (Table 2). Although linolelaidic acid was detectably found to exist in perfusate triacylglycerol at $1 \mathrm{~h}$ of the perfusion period, it gradually decreased thereafter during the entire experimental period in control livers. Also, no cumulative increase in the secretion of triacylglycerol enriched with endogenous fatty acids was observed during the entire experimental period in control liver. The addition of emeriamine at $2 \mathrm{~h}$ caused an immediate and marked increase in the secretion of linolelaidate as triacylglycerol into liver perfusate (4.3-fold at $3 \mathrm{~h}$ and 10 -fold at $4 \mathrm{~h}$, compared with the value at $2 \mathrm{~h}$ ). This drug also caused an increased secretion of endogenous fatty acids as perfusate triacylglycerol ( 1.5 -fold at $3 \mathrm{~h}$ and 2.4 -fold at $4 \mathrm{~h}$, compared with the value at $2 \mathrm{~h}$ ), but the extent of the increases was more moderate than that of exogenous fatty acid.

In contrast to the situation in triacylglycerol secretion, cholesterol was continuously accumulated in the perfusion medium during the entire experimental period, even in the control liver. Emeriamine added at $2 \mathrm{~h}$ of the experimental period magnified the extent of cholesterol accumulation in liver perfusate. Consequently the drug treatment significantly increased the amount of cholesterol secreted by the liver at $3 \mathrm{~h}$ and $4 \mathrm{~h}$ of the experimental period. The extent of the increase by emeriamine was, however, much less in cholesterol secretion than in triacylglycerol secretion.

\section{Incorporation of exogenous transfatty acid into various lipids}

As shown in Table 3, the drug treatment caused a marked increase in the proportion of transfatty acid in hepatic triacylglycerol. The proportion of transfatty acid in triacylglycerol was, however, significantly lower in the liver than in perfusate for the control and the drug-treated groups $(p<0.05)$. The percentages of exogenous transfatty acid in cholesterol ester and phospholipid in perfusates and postperfused livers of control rats were very low. However, an inhibition of fatty acid oxidation by emeriamine was associated with a concomitant increase in the incorporation of exogenous transfatty acid into perfusate and hepatic phospholipid. The drug also caused a marked increase in the proportion of transfatty acid in cholesterol ester in perfusate, but not in postperfused liver.

\section{DISCUSSION}

This study was carried out to examine the relative significance of the endogenous- and/or exogenous-free fatty acids as the substrate for the increased synthesis and secretion of lipids, especially triacylglycerol, by the 2-d fasting rat liver following inhibition of fatty acid oxidation by emeriamine, a specific inhibitor of liver mitochondrial carnitine palmitoyltransferase I. For this purpose, we 
Effect of Emeriamine in Perfused Rat Liver

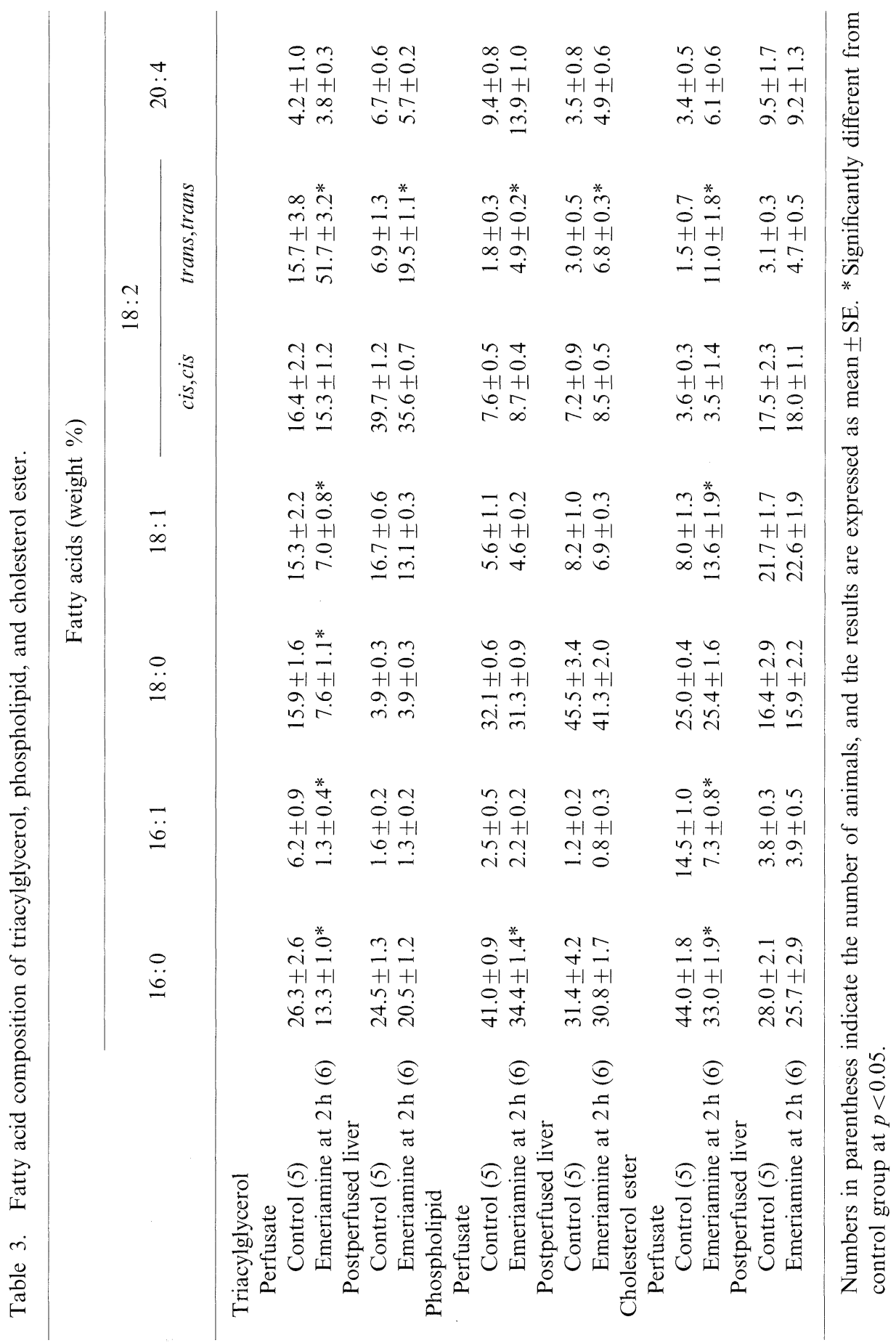

Vol 44, No 4, 1998 
employed a geometrical isomer of linoleic acid, 9,12-trans,trans-octadecadienoic acid (linolelaidic acid), as a tracer to examine the metabolic fate of exogenous fatty acid. The reported data suggested that metabolic rate in oxidation and esterification pathways are somewhat different between trans- and cis-fatty acids $(9,10,18)$. This di-transfatty acid used in the present study, however, is still advantageous, since this type of transfatty acid is easily distinguishable from endogenous fatty acids by gas liquid chromatography $(9,10,18)$. Thus we could compare the relative importance of the endogenous and exogenous fatty acids for the synthesis and secretion of lipids, especially triacylglycerol.

Consistent with previous liver perfusion studies using oleic acid substrate (6), the present study again showed that the addition of emeriamine causes complete cessation of ketone body production by the 2-d fasting rat livers perfused with linolelaidic acid substrate. Kanamaru et al reported that emeriamine inhibits carnitine-dependent oxidation to ${ }^{14} \mathrm{CO}_{2}$ of $1-{ }^{14} \mathrm{C}$-palmitate in a dose-dependent manner, but it has little effect on the oxidation to ${ }^{14} \mathrm{CO}_{2}$ of $1-{ }^{14} \mathrm{C}$-palmitoylcarnitine and $1-{ }^{14} \mathrm{C}$-octanoate in hepatic mitochondria isolated from a fasting rat $(3,5)$. Harano et al also reported that emeriamine inhibits the incorporation of $\mathrm{U}-{ }^{14} \mathrm{C}$-palmitate into acid-soluble fractions (ketone bodies) in hepatocytes isolated from a fasting rat, and further showed that this drug exerts inhibitory effect on mitochondrial outer membrane carnitine palmitoyltransferase I (4). In the present study, we confirmed that emeriamine inhibits carnitine palmitoyltransferase activity in perfused liver (Table 1). These observations therefore suggest that the decreased rate in transportation of endogenous and exogenous fatty acids across the mitochondrial membrane through the inhibition by emeriamine of carnitine palmitoyltransferase causes the cessation of ketone body production as observed in the present study.

The ratio of $\beta$-hydroxybutyrate to acetoacetate was decreased rapidly following emeriamine treatment. Because it has been reported that this oxidation-reduction couple reflects the mitochondrial oxidation-reduction equilibrium (19), the decreased ratio represents a shift toward a more oxidized state of the mitochondrial compartment as a result of the decreased incorporation of endogenous and exogenous fatty acids into mitochondria for fatty acid oxidation and thus ketone body production (Table 2 ).

Control livers from 2-d fasting rats secreted triacylglycerol until $1 \mathrm{~h}$, but not thereafter (Table 2). Furthermore, the accumulation of exogenous transfatty acid in secretory triacylglycerol was only $16 \%$ at the end of the perfusion period; the remaining $84 \%$ of the fatty acids was derived from endogenous origins. This result suggests that the contribution of endogenous fatty acids, probably arising from the catabolism of hepatic lipids, is much greater than the exogenous substrates for the synthesis and secretion of triacylglycerol in the fasting rat liver. On the other hand, McGarry \& Foster and Ide \& Ontko reported that the marked increase in the production of ketone bodies from exogenous ${ }^{14} \mathrm{C}$-oleic acid by the liver from fasting rats was accompanied by a concomitant depression of the incorporation of exo- 
genous fatty acids into the esterification pathway, especially for the synthesis of triacylglycerol $(20,21)$. Moreover, Ide and Ontko reported that the contribution of exogenous fatty acid can be more than $80 \%$ of total ketone body production (21). Therefore the impaired triacylglycerol secretion and the reduced incorporation of exogenous transfatty acid into triacylglycerol molecule by the livers of fasting rats may be caused by a depletion of exogenous fatty acids available for the synthesis and secretion of triacylglycerol through the stimulation of fatty acid oxidation, but not by any defects involved in the esterification pathway.

The inhibition of fatty acid oxidation by emeriamine increased the proportion of linolelaidic acid accompanying the concomitant decreases in the proportions of endogenous fatty acids such as palmitic, palmitoleic, stearic, and oleic acids, but not linoleic acid in perfusate triacylglycerol (Table 2). Furthermore, discrimination by gas liquid chromatography of endogenous fatty acids from exogenous fatty acids can allow us to estimate that exogenous fatty acid compared with the endogenous fatty acid serves as the preferred substrate for the formation and secretion of triacylglycerol-rich lipoproteins when the fasting rat livers have been treated with fatty acid oxidation inhibitor. Fatty acid analyses also showed that the inhibition of fatty acid oxidation by emeriamine diverts exogenous fatty acid primarily to triacylglycerol and to a lesser extent to cholesterol ester and phospholipid (Table 3 ), consistent with a previous study using 2-tetradecylglycidic acid (TDGA) as an inhibitor of fatty acid oxidation (21).

Emeriamine treatment increased the proportions of exogenous transfatty acid in triacylglycerol and cholesterol ester, but not in phospholipid, more in perfusate than in the postperfused liver, suggesting that free fatty acid supplied exogenously during the perfusion periods incorporates into hepatic triacylglycerol and cholesterol ester, and it is preferentially secreted in the form of lipoprotein instead of being stored in the liver.

The mechanisms responsible for an increased hepatic secretion of cholesterol following inhibition of fatty acid oxidation remain obscure. It has been reported that impairment of fatty acid oxidation at the step of carnitine palmitoyltransferase by the inhibitors, such as carnitine analogues or TDGA, greatly stimulated the cholesterol-synthesizing capacity of the liver, which is accompanied by an increased secretion of cholesterol by the liver $(21,22)$. Goh and Heimberg also reported that increased secretion of triacylglycerol following the infusion of exogenous oleic acid resulted in a simultaneous enhancement of the activity of hepatic cholesterol synthesizing enzymes and the secretion of cholesterol $(23,24)$. It is therefore likely that the increased hepatic secretion of cholesterol following inhibition of fatty acid oxidation by emeriamine is, in part, attributed to an enhancement of the cholesterol synthesis de novo in the liver, although the effect of emeriamine on hepatic cholesterol synthesis remains to be investigated.

In summary, we demonstrated again that the fatty acid oxidation rate is crucial for the synthesis and secretion of triacylglycerol-rich lipoproteins in rat liver. 


\section{REFERENCES}

1) Heimberg M, Goh EH, Klausner HA, Soler-Argilaga C, Weinstein I, Wilkox HG. 1978. Regulation of hepatic metabolism of free fatty acids: Interrelationships among secretion of very-low-density lipoproteins, ketogenesis, and cholesterogenesis. In: Disturbances in Lipid and Lipoprotein Metabolism (Dietschy JM, Gotto AM, Ontko JA, eds), p 251-267. Williams \& Wilkins, Baltimore.

2) McGarry JD, Foster DW. 1980. Regulation of hepatic fatty acid oxidation and ketone body production. Ann Rev Biochem 49: 395-420.

3) Kanamaru T, Shinagawa S, Asai M, Okazaki H, Sugiyama Y, Fujita T, Iwatsuki H, Yoneda M. 1985. Emeriamine, an antidiabetic $\beta$-aminobetaine derived from a novel fungal metabolite. Life Sci 37: 217-223.

4) Harano Y, Kojima H, Kashiwagi A, Tanaka Y, Nakajima T, Fujita T, Shigeta Y. 1987. A new carnitine analogue for the correction of metabolic derangements in diabetes. In: Recent Trends in Management of Diabetes Mellitus (Sakamoto N, Alberti KGMM, Hotta N, eds), p 567-571. Elsevier Science Publishers, New York.

5) Kanamaru T, Okazaki H. 1989. Emeriamine: a new inhibitor of long chain fatty acid oxidation and its antidiabetic activity. In: Novel Microbial Products for Medicine and Agriculture (Demain AL, Somkuti GA, Hunter-Cevera JC, Rossmoore HW, eds), p 135-144. Society for Industrial Microbiology, Elsevier, New York.

6) Yamamoto K, Fukuda N, Fukui M, Kai Y, Ikeda H, Sakai T. 1996. Increased secretion of triglyceride and cholesterol following inhibition of long-chain fatty acid oxidation in rat liver. Ann Nutr Metab 40: 157-164.

7) Maeda H, Fujiwara M, Fujita K, Fukuda N. 1996. Hypertriglyceridemia and fatty liver of fasting rats after administration of emeriamine. J Nutr Sci Vitaminol 42: 111120.

8) Maeda H, Fujiwara M, Miyamoto K, Hamamoto H, Fukuda N. 1996. Effects of refeeding diets on emeriamine-induced fatty liver in fasting rats. $J$ Nutr Sci Vitaminol 42: 469-478.

9) Fukuda N, Igari N, Etoh T, Hidaka T, Ikeda I, Sugano M. 1993. A comparison of the metabolism of cis,cis-, cis,trans/trans, cis- and trans,trans-9,12-octadecadienoic acids in rat liver. Nutr Res 13: 779-786.

10) Fukuda N, Etoh T, Wada K, Hidaka T, Yamamoto K, Ikeda I, Sugano M. 1995. Differential effects of geometrical isomers of octadecadienoic acids on ketogenesis and lipid secretion in the livers from rats fed a cholesterol-enriched diet. Ann Nutr Metab 39: 185-192.

11) Fukuda N, Azain MJ, Ontko JA. 1982. Altered hepatic metabolism of free fatty acids underlying hypersecretion of very low density lipoproteins in the genetically obese Zucker rat. J Biol Chem 257: 14066-14072.

12) Fukuda N, Ontko JA. 1984. Interactions between fatty acid synthesis, oxidation, and esterification in the production of triglyceride-rich lipoproteins by the liver. $J$ Lipid Res 25: 831-842.

13) Yamamoto K, Fukuda N, Shiroi S, Shiotsuki Y, Nagata Y, Tani T, Sakai T. 1994. Effects of dietary fat levels on the absorption and tissue accumulation of probucol in the rat. Arzeim.-Forsch/Drug Res 44(II): 1059-1062.

14) Markwell MAK, McGroarty EJ, Bieber LL, Tolbert NE. 1973. The subcellular distribution of carnitine acyltransferases in mammalian liver and kidney. $J$ Biol Chem 248: 3426-3432. 
15) Lowry OH, Rosebrough HJ, Farr AL, Randall RJ. 1951. Protein measurement with the folin phenol reagent. J Biol Chem 193: 265-275.

16) Folch J, Lees M, Sloane-Stanley GH. 1957. A simple method for the isolation and purification of total lipids from animal tissues. $J$ Biol Chem 226: 497-509.

17) Snedecor GW, Cochran WG. 1967. Statistical Method, 6th ed, p 258-338. Iowa State University Press, Ames, Iowa.

18) Ide T, Sugano M. 1984. Oxidation and esterification of cis- and trans-isomers of octadecenoic and octadecadienoic acids in isolated rat liver. Biochim Biophys Acta 794: 281-291.

19) Wilson DF, Stubbs M, Veech RL, Ercinska M, Krebs HA. 1974. Equilibrium relations between the oxidation-reduction reactions and the adenine triphosphate synthesis in suspensions of isolated liver cells. Biochem J 140: 57-64.

20) McGarry JD, Foster DW. 1971. The regulation of ketogenesis from oleic acid and the influence of antiketogenic agents. $J$ Biol Chem 246: 6247-6253.

21) Ide T, Ontko JA. 1981. Increased secretion of very low density lipoprotein triglyceride following inhibition of long chain fatty acid oxidation in isolated rat liver. $J$ Biol Chem 256: $10247-10255$.

22) Jansen H, Hoogerbrugge NL, Hulsmann WC. 1990. Inhibition of carnitinepalmitoyltransferase leads to induction of 3-hydroxymethylglutaryl coenzyme A reductase activity in rat liver. Biochim Biophys Acta 1044: 390-393.

23) Goh EH, Heimberg M. 1976. Effect of oleic acid and cholesterol on the activity of hepatic hydroxymethylglutaryl coenzyme A reductase. FEBS Lett 63: 209-210.

24) Goh EH, Heimberg M. 1977. Effects of free fatty acids on activity of hepatic microsomal 3-hydroxy-3-methylglutaryl coenzyme A reductase and on secretion of triglyceride and cholesterol by the liver. $J$ Biol Chem 252: 2822-2826. 